\title{
Design of Integrated Machine for Community Science Popularization
}

\author{
Qian Zhang ${ }^{\mathrm{a}}$, Feng $\mathrm{Yu}^{\mathrm{b}}$, Rong $\mathrm{Fu}^{\mathrm{c}}$, Xin Liu ${ }^{\mathrm{b}}$, Junfeng Zhang ${ }^{\mathrm{b}}$ \\ ${ }^{1}$ Institute of Agricultural Scientech Information, Beijing Academy of Agriculture and Forestry \\ Sciences, Beijing, 100097, China \\ aemail: zhangqianshuxue@126.com, bemail: yuf@agri.ac.cn, email: fur@agri.ac.cn, \\ demail: liux@agri.ac.cn, eemail: zhangjf@agri.ac.cn
}

Keywords: Integrated Machine; Community Science Popularization; Information Services

\begin{abstract}
A kind of integrated machine was designed for community science popularization. It was built rich digital science resources, and information resource was customizable, updated quickly and targeted. So as to meet the individual needs of community residents. The integrated machine used touch screen, and easy to use, easy to operate and highly interactive. It was able to pass scientific knowledge to the majority of community residents vividly, by video, pictures, text and other diverse forms.
\end{abstract}

\section{Introduction}

With the development of China's economic and adjustment between urban and rural, urban residents became more and more. The importance of community science popularization became increasingly prominent. At present, the majority of urban residents of China still needed low-cost, simple type information products and services [1]. It was a new facing problem after solving science popularization "last kilometer".

Research found that the conventional mass media always with single and boring disseminate content. Computers, mobile phones and other new equipments were able to meet the demand of multimedia distribution, but some older residents were unskilled operation of these devices [2]. Although there were a variety of science popularization instruments, they all had some disadvantages: The traditional means were always with small amount of information, time-consuming and labor-intensive; Modern means were limited by the equipment and operational skills. It is in urgent need of a new way of community science popularization, which is efficient, convenient, easy to install and highly interactive.

\section{Integrated Machine for Science Popularization}

Integrated machine for science popularization service in the community was combined with computer and touch-screen integrated machine [3] [4]. It was with vertical and dual screen (touch screen and display screen) set, which set computer access, information services, distance education, telephone consultation, video interactive, communication and other functions in one. With the camera assembled in the integrated machine users could chat with remote experts "face to face" in real time. Without keyboard and mouse, users just tap the touch screen with fingers would be able to browse information on the internet, read e-books, demand video courseware, discover science information as well as learn agricultural market and so on. The integrated machine realized "unified collection, management and service" of information resources, and provided scientific, efficient and systematic information services for the public [5].

Integrated machine for science popularization service in the community was developed with B/S architecture, which with five layers: user layer, presentation layer, logic layer, data layer and system layer. The user level browsed and inquired information resource by the integrated machine [6]. The presentation layer, with the logic layer support, called the appropriate information section and application services according to individual needs of different clients. The logic layer was the core part, which applied and set parameters of different functional modules to complete the application processing tasks and achieve functional. The data layer was on the ground floor, which processed 
the data requests of logic layer through the data interface. The system layer was mainly used for data storage support of system's support and stability. The whole system was based on the windows platform, and used .net and C \# developed language. The overall architecture of the system was shown as in Figure 1.

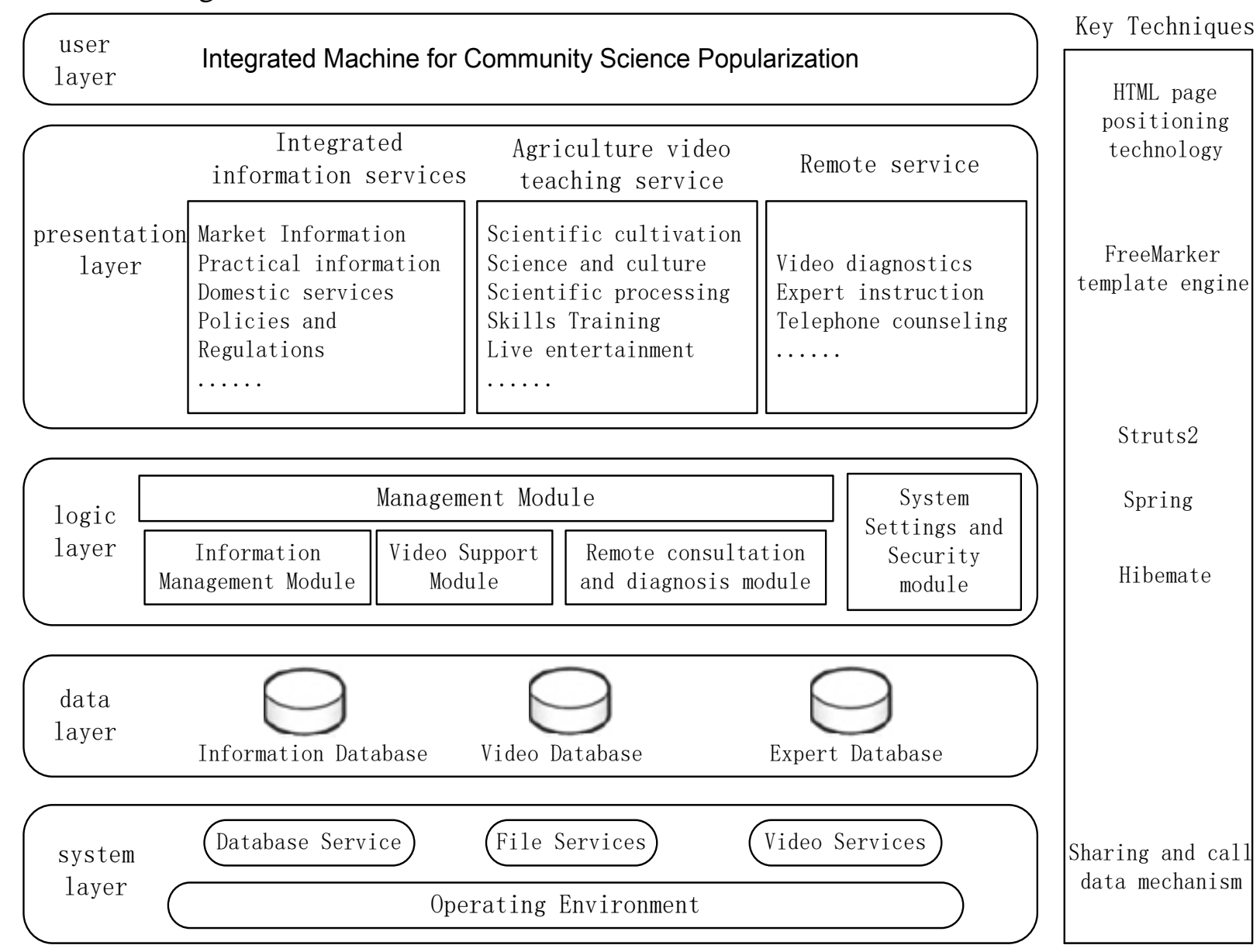

Fig.1. The architecture of integrated machine for science popularization

Integrated machine for science popularization service, around the needs of the people, provided efficient, convenient, simple intuitive and interaction science popularization service with the use of use of modern information technology. The machine used streaming media technology and compatible with the current mainstream media formats, so as to users could online viewing and leave messages. By the use of audio and video equipment, the integrated machine could achieve real-time online remote interaction, play teaching video and online Q \& A. Rely on multi-touch technology, users could use intuitive finger operation (such as drag, distraction, close up and rotation) to switch, shift, zoom, flip and rotate pictures, documents and web pages, as well as play videos. In addition, those residents who have never used a computer can also do various operations and get information according to the operation tips on touch-screen.

Administrators of the integrated machine for science popularization could submit, modify, approve, publish and push the information resources of the system. The management platform of the integrated machine could handle data in various formats and achieved automation and standardization, which was enter convenient, fast and friendly interface. The core administrator managed the system overall, who had the highest level of management authority and could real-time monitoring the operation of integrated machine. The data resources of the whole system could be backed up regularly and could also be recovered when necessary. Simultaneously, the system was also able to manage system running log and user activity log. The data resources of the machine mainly included scientific and technological information, science education data, self-distribution system data, manually adding data and some local educational video and animation resources. In order to reduce the data maintenance, in addition to locally added data was stored in a local database, the rest of the data was all called form other library data through the interfaces. 


\section{Data Management Platform.}

The Data management platform was able to unified manage many integrated machine display terminal. Just one management platform could control a plurality of display terminals, and achieved “a key update, multi-point release". The platform mainly achieved integration and management of background data of system. Its key features included: data management, category management, data push, remote monitoring, user management, log management, statistical analysis, smart upgrade and other functional modules. The entire platform was designed by B / S architecture. The data interface module on server-side called data using $\mathrm{C} \#$ and Web Service. The monitoring module was developed with $\mathrm{C} \#$ language and was responsible for monitoring the switch, log length, smart upgrade and other operating conditions of the machine.

\section{Information Display Platform.}

The platform was installed in each touch-screen terminal device, and mainly achieved the presentation, management and update of the built resource in the device. The platform was with the main achievement of information display, data transmission, video diagnostics, data call, message notification and other modules. The client data show module, by custom developed browser housing embedded flash guide page, had better page display.

\section{Community Science Popularization Service}

The integrated machine combined video, network, 3G, GPRS, intelligent decision, XMPP and a variety of high-tech in one, and realized online promotion of scientific and technological, science popularization, policies and regulations, animation science, leisure agriculture and other information [7] [8]. It also achieved audio and video interactive between experts and users, intelligence consulting, information push and other functions. All in all, there were great advantages of the integrated machine in the community science popularization service.

\section{Powerful Digital Asset Management.}

The management platform was designed with .net technology and B / S architecture, which were able to achieve unite Integration, manufacture and management of the information content in the integrated machine. It also achieved uploading and downloading of large files, storage management of massive Data, and tacked ease use and security into account. The platform was scalability and easy integrated a variety of technology and resources, which achieved distributed control, stability and versatile. Currently, the technology application and the integrated data resources of the platform have reached advanced domestic level.

\section{Personalized Community Science Popularization.}

By field researched the selected streets, communities, villages and towns and other service agencies, we have a good grasp of the different needs of residents in all levels. So as to the construction of data resource in the integrated machine has scientific evidence, and could meet community residents' diverse science popularization needs. The platform intelligent recommended and adjusted the related information display by analyses user habit, and then, to provide more convenient and intelligent science popularization services for user and more personalized services for local community residents.

\section{Abundant form of Community Science Popularization.}

The users were able to enjoy entertainment program, playing games and online Interactive through the integrated machine, which would enhance comprehensive cultural quality of residents and rich cultural life of residents in the entertaining form. Simultaneously, when the residents encounter problems in production and life, they could real-time video call with agricultural experts and solve technical problems. It helped community residents solve the problem timely, expanded channels of access information, and was beneficial to enhance the overall quality of residents.

\section{Conclusion}

The integrated machine for community science popularization was a necessary and benefit supplementary for quality training, science popularization and integrated information services for 
community residents. At the same time, it was also an attempt and innovation for a new type of training model, which has important role and significance and also be a trend. The integrated machine will be developed smarter and more convenient in the future. Its onboard content will be more rich and practical, user experience will focus more on interactive, touch screen interface will be clearer, and the whole function will be more powerful.

\section{Acknowledgement}

In this paper, the research was sponsored by 2015 Beijing Fund for Agriculture (project of "Development and application of 'Beijing agricultural technician' micro-channel service platform").

\section{References}

[1] Yexian Long, Bo Gao, Guoping Zeng. On the Residents Subjectivation Shift of Science and Technology Popularization Model [J], Study on Science Popularization, 2012 (4): 11-15.

[2] Junping $\mathrm{Hu}$, Shunke Shi. A Study on the Citizen's Needs and Satisfaction of Science Popularization in the Urban Community of China [J], Study on Science Popularization, 2010 (5): 18-26.

[3] Ruifeng Kang. Smart PCTV Program Based on Touch-screen of Android 4.X [J], Internet of Things Technology, 2013 (10): 61-63, 67.

[4] Ruifeng Kang. Discussion on Technical Solutions of Touch-screen of Android 4.X [J], Wireless Internet Technology, 2013 (1): 166-167.

[5] Xiaofeng Jiang, Weifeng Shi, Yijian Liu and so on. Monitoring and Control System Based on Touch Screen and PLC for Shipboard Power Station [J], Electric Power Automation Equipment, 2011, 31 (1): 122-125.

[6] Chao Zhou, Chen Wang, Yanjun Fang. Design of LCD Control and Touch Screen Interface Based on W77E58 [J], Instrument Technique and Sensor, 2009 (3): 70-71, 74.

[7] Youwen Tian, Xiaojuan Wang. Design of Hand-held Leaf Area Meter Based on ARM9 [J], Journal of Agricultural Mechanization Research, 2009 (1): 149-151.

[8] Nan Xue, Wei Qiao. The thinking of Lenovo Group Developed Intelligent TV Industry [J], Chinese High-tech Enterprises, 2012 (11): 16-17. 